This document is the Accepted Manuscript version of a Published Work that appeared in final form in Accounts of Chemical Research 52(3) : 686-694 (2019), copyright (C) 2019 American Chemical Society after peer review and technical editing by the publisher. To access the final edited and published work see https://doi.org/10.1021/ acs.accounts.8b00566

\title{
Innovative Electrolytes Based on Ionic Liquids and Polymers for Next- Generation Solid-State Batteries
}

\author{
Maria Forsytha, ${ }^{\mathrm{a}, \mathrm{b}, \mathrm{c}}$, Luca Porcarellia, ${ }^{\mathrm{a} b}$, Xiaoen Wang $^{\mathrm{a}}$, Nicolas Goujon ${ }^{\mathrm{a}}$, and David Mecerreyes ${ }^{\mathrm{b}}$ \\ Institute for Frontier Materials, Deakin University, Geelong, VIC 3217, Australia \\ Polymat, Institute for Polymer Materials, University of the Basque Country UPV/EHU, Joxe Mari Korta Center, Avda. \\ Tolosa 72, 20018 Donostia-San Sebastian, Spain \\ ARC Centre of Excellence for Electromaterials Science (ACES), Deakin University, Burwood, VIC 3125, Austrailia \\ *Corrisponding author E-mail: maria.forsyth@,deakin.edu.au
}

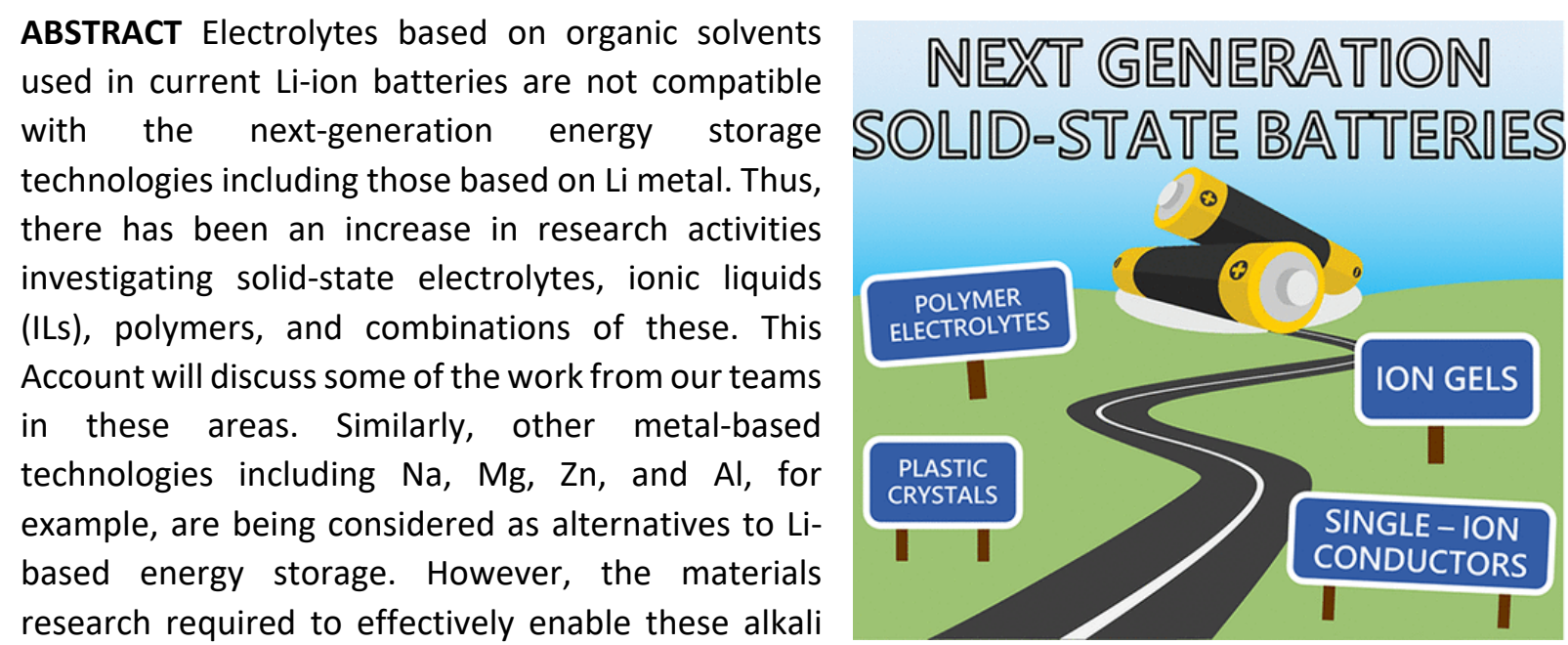
metal based energy storage applications is still in its relative infancy. Once again, electrolytes play a significant role in enabling these devices, and research has for the most part progressed along similar lines to that in advanced lithium technologies. Some of our recent contributions in these areas will also be discussed, along with our perspective on future directions in this field. For example, one approach has been to develop single-ion conductors, where the anion is tethered to the polymer backbone, and the dominant charge conductor is the lithium or sodium countercation. Typically, these present with low conductivity, whereas by using a copolymer approach or incorporating bulky quaternary ammonium co-cations, the effective charge separation is increased thus leading to higher conductivities and greater mobility of the alkali metal cation. This has been demonstrated both experimentally and via computer simulations. Further enhancements in ion transport may be possible in the future by designing and tethering more weakly associating anions to the polymer backbone. The second approach considers ion gels or composite polymer electrolytes where a polymerized ionic liquid is the matrix that provides both mechanical robustness and ion conducting pathways. The block copolymer approach is also demonstrated, in this case, to simultaneously provide mechanical properties and high ionic conductivity when used in combination with ionic-liquid electrolytes. The ultimate electrolyte material that will enable all high-performance solid-state batteries will have ion transport decoupled from the mechanical properties. While inorganic conductors can achieve this, their rigid, brittle nature creates difficulties. On the other hand, ionic polymers and their composites provide a rich area of chemistry to design and tune high ionic conductivity together with ideal mechanical properties. 


\section{Introduction}

As we catapult toward the end of the second decade of the 21st century, the demand for clean, more reliable, and globally affordable energy to power our factories, our homes, and our transportation is growing. Furthermore, the thirst for new, energy-hungry technologies such as ever more complex portable electronics, drones, etc. results in an ever-increasing drive for new materials and new knowledge to create future energy storage solutions. The imperatives here will often involve the competing requirements of safety, cost, energy density, power density, ethical sourcing, and security of supply as well as the move toward designing for a circular economy, i.e., repurposing or recycling of materials and devices. $(1,2)$

Lithium-ion technologies based on a variety of different chemistries have dominated the research arena, and now, the market share of energy storage; however, they are unable to satisfy all requirements and are likely to be augmented, if not replaced, in the longer term by a series of other technologies designed for particular applications. For example, Li metal offers the hope of higher energy density, especially when coupled with cathodes such as sulfur, air, or high-nickel-contentlayered oxides. Electrolytes based on organic solvents are not compatible in these cases, spurring the increase in research into solid-state electrolytes, ionic liquids, polymers, and their combinations. Some of our recent work in these areas will be discussed along with the future outlook in this field.

Apart from inorganic ceramic or glassy electrolytes,(3) recent electrolyte materials that have shown potential in beyond Li-ion energy storage technologies can be categorized as follows: solid polymer electrolytes (SPEs),(4) ionic liquids, gels, and nanocomposites,(5) and organic ionic plastic crystals.(6) The resurgence in SPE research has arisen from the impetus for safer technologies and, in particular, Li metal and the recognition that many applications benefit from elevated temperatures $\left(50-100^{\circ} \mathrm{C}\right)$ rather than room temperature. Indeed, current Li-ion technologies require sophisticated cooling and control modules to ensure that the operating temperature is kept below $60^{\circ} \mathrm{C}$. Moreover, the battery performance and life are seriously compromised if the temperature is regularly above room temperature. $(7,8)$ Thus, future electrolyte research will not be restricted to room-temperature conductors. Ionic liquids offer many desirable and tailorable features as electrolytes in high-energydensity batteries. For example, they can have large electrochemical windows that enable metal anodes and high-voltage cathodes, they can have minimal volatility and zero flammability, and they have higher temperature stability.(9) On the other hand, polymers have been proposed for all solidstate batteries since the mid-1970s when ion conductivity was discovered in polyether-salt complexes. $(10,11)$ Combinations of ILs and polymers have also been considered for some time, especially in the form of gel electrolytes. $(12,13)$

Polymer electrolytes are commonly formed by a mixture of an alkali metal salt with an ion-solvating polymer host, such as poly(ethylene oxide) (PEO). Two main ion transport mechanisms take place in polymer electrolytes: (1) diffusion via a hopping mechanism between ion coordination sites through structural rearrangements or (2) segmental motion of the coordination sites on the polymer backbone. The first mechanism, also classified as Grotthus-like, follows an Arrhenius dependence on temperature and occurs in highly concentrated systems.(5) This mechanism is often decoupled from the bulk dynamics of the electrolyte. On the other hand, VFT behavior occurs when ion dynamics rely on polymer segmental motion, with a lower Tg leading to higher conductivity.

Regardless of the transport mechanism, much of the ionic current is usually transported by anions rather than metal cations such as in the PEO-salt electrolytes. Consequently, severe concentration gradients form during operation that eventually lead to poor cell performance of polymer electrolytes. 
Different strategies have been investigated to increase the cation transport number $(>0.5)$ and overcome this limitation.(4) Our recent work on this aspect will be discussed in the following section.

\section{Anionic Single-Ion Conductors}

The movement of anionic charges can be restricted by attaching the anions to the polymer backbone so that the cation is the only ion free to move. For this reason, these polymer electrolytes are known as single-ion conductors, as they are characterized by a cation transport number virtually equal to unity. As evidenced by the recent review of Armand and coauthors,(14) the single-ion conductors display ionic conductivity generally lower than that of their dual-ion counterparts. In the following section of this Account, two main strategies to increase the ionic conductivity of single-ion conductors will be discussed, and we will present examples of these based on our long-standing interest in singleion conductor systems. The first strategy involves the design of systems with a low glass transition temperature and increased polymer segmental mobility. The second approach decouples the ionic conductivity from the dynamics of the polymer backbone.

\subsection{Copolymerization Approach To Increase the Segmental Mobility Dynamics}

A large number of anionic polymers are today available as single-ion conductors for lithium and sodium. In our work, we focused on vinyl-, acrylamide-, and methacrylate-based single-ion conductors. We chose radical polymerization techniques as opposed to polycondensation and polyaddition because of affordability and simplicity as well as the high tolerance of radical polymerization toward ionic functional groups. The general chemical structures of these polymers are shown in Figure 1a.

(a)

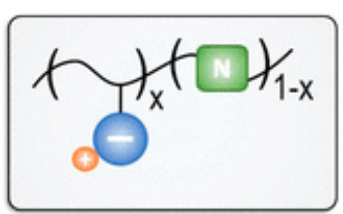

Single-ion "copolymer" conductors

(b)

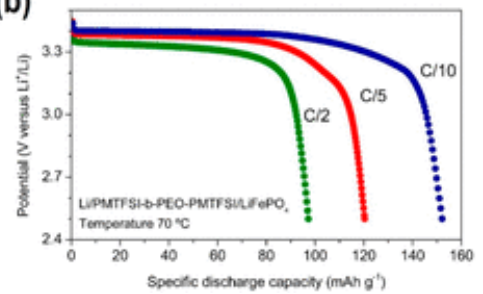

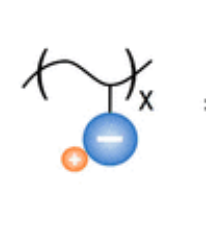

(c)

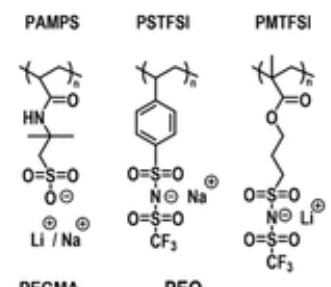

PEGMA PEO
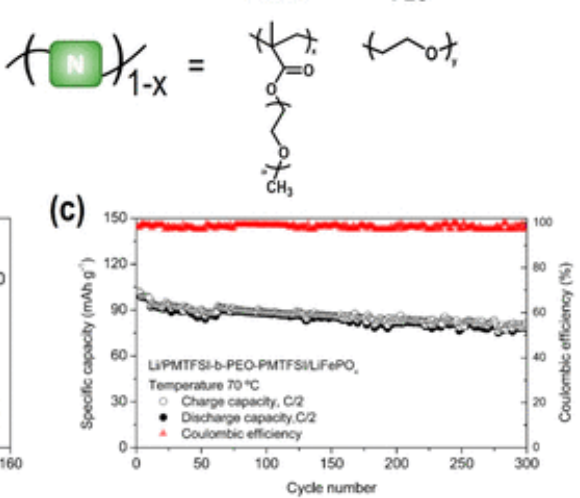

Figure 1. (a) Structures of the single-ion copolymers described in this Account, (b) discharge profiles, and (c) cycling performances of Li metal/PMTFSI-b-PEO-b-PMTFSI)/LiFePO4. Adapted with permission from ref (15). Copyright 2017 Elsevier.

In 2016, we developed single-ion conductors based on poly(lithium 1-[3-(methacryloyloxy)propylsulfonyl]-1-(trifluoromethylsulfonyl)imide) (PMTFSI). The homopolymers of PMTFSI demonstrated a high glass transition temperature $\left(\mathrm{Tg}>90^{\circ} \mathrm{C}\right)$ and, consequently, low-roomtemperature conductivity; thus, we introduced pendant ethylene oxide units with the aim to increase the flexibility of the polymer backbone (Figure 1a). When LiMTFSI was copolymerized with 
poly(ethylene glycol) methyl ether methacrylate (PEGM), the $\mathrm{Tg}$ decreased well below room temperature, and conductivity increased up to $2.3 \times 10-6 \mathrm{~S} \mathrm{~cm}-1$ at $25^{\circ} \mathrm{C}$.(16) The presence of a single Tg demonstrated a good miscibility of the PEGM with LiMTFSI. Despite the improvement in ionic conductivity, the mechanical properties of these polymers decreased, and we were unable to prepare freestanding films.(16)

In order to improve the mechanical properties, we prepared single-ion triblock copolymer electrolytes based on linear PEO and side PMTFSI blocks, PMTFSI-b-PEO-b-PMTFSI, controlling PEO crystallinity by changing the composition of the block copolymers. This facilitated highly conductive and freestanding films with a lithium transference number close to unity. PMTFSI-b-PEO-b-PMTFSI electrolytes showed excellent performance in LiFePO4 metal polymer cells operating at $70{ }^{\circ} \mathrm{C} .(15)$ (Figure 1b,c)

It is known that small organic molecules can be used as a plasticizer for the polymer backbone in order to decrease the $\mathrm{Tg}$ and increase the conductivity of single-ion conductors. Very recently, we prepared cross-linked electrolytes by copolymerization of PEG dimethacrylate (PEGDM), PEGM, and LiMTFSI in the presence of propylene carbonate. Besides plasticizing the polymer, the high dielectric constant of propylene carbonate assists the dissociation of the lithium cations and the covalently bonded anions, thus increasing the fraction of mobile ions. This material displayed a unique combination of high lithium transference number and high conductivity at ambient temperature $(\sigma \approx 10-4 \mathrm{~S} \mathrm{~cm}-1)$. The cross-linked network showed excellent performances in LiFePO4 metal polymer cells at room temperature.(17) As a drawback, this material includes a flammable organic solvent, which we ideally could eliminate from future devices.

\section{2. "Mixed" Co-Cation Approach To Decouple Li/Na-ion Transport from Segmental Mobility}

An alternative approach is to design polymer electrolytes such that ions are mobile even below the glass transition temperature, therefore decoupling the polymer segmental motion from ionic conductivity. Initially, we selected poly(2-acryl-amido-2-methyl-1-propane-sulfonate) homopolymer (PAMPS) as a polymer backbone for sodium single-ion conductors. The work of Colby and co-workers serving as inspiration,(18) a number of bulky quaternary ammonium cations replaced sodium ions in the polymer backbone; their chemical structures are shown in Figure 2.

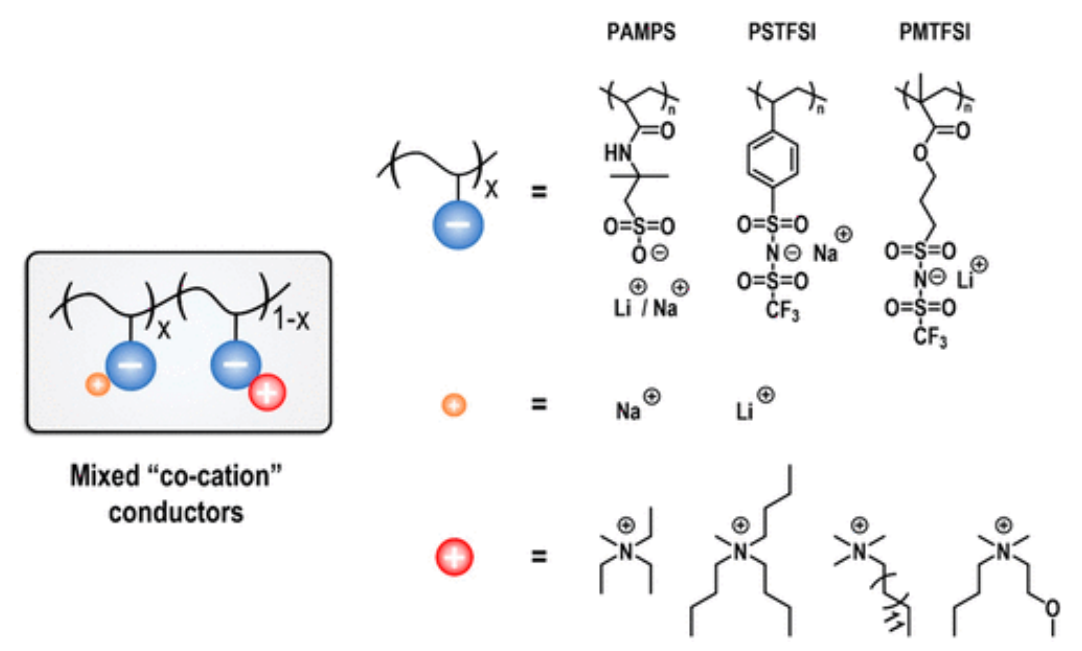

Figure 2. Schematic of a mixed co-cation conductor and the chemical structures of the bulky quaternary ammonium cations described in this Account. 
Significant ionic conductivities were measured below the $\mathrm{Tg}$ of the polymer; the highest conductivity was found for the system containing $10: 90 \%$ mol ratio of Na+/triethylmethylammonium (N1222) cations.(19) We hypothesize that the addition of bulky cations can increase the polymer free volume and "loosen" the structure to encourage metal cation hopping, facilitated by the charged backbone. Subsequently, we extended our interest to different polymer backbones including the copolymer of PAMPS with poly vinyl sulfonate (PVS)(20) and the copolymer of polystyrenesulfonyl(trifluoromethylsulfonyl)imide with ethyl acrylate.(21) We observed similar evidence of decoupling in all of these systems. However, the ionic conductivities measured in these systems were still too low for application in practical devices. The addition of a small amount of a tetraglyme plasticizer resulted in an increase of ionic conductivity of several orders of magnitude, up to $\sim 10-5 \mathrm{~S} \mathrm{~cm}-1$ at $50^{\circ} \mathrm{C} .(19,20)$

Following this approach, we prepared PAMPS polymers containing lithium cations mixed with N1222 or dimethylbutylmethoxyethylammonium (N114(201)) cations.(22) Again, the temperature dependence of the conductivities for both samples appeared to be independent of Tg. Moreover, $7 \mathrm{Li}$ NMR line-width analysis suggested that ion hopping was the predominant mechanism for lithium diffusion for this polymer system. In contrast to sodium-based ionomers, $(19,20)$ the incorporation of small amounts of tetraglyme did not increase the ionic conductivity. We hypothesized that the strong interactions between lithium ions and ether oxygen atoms may cause an unexpected recoupling of the lithium diffusion dynamics with the polymer segmental motion,(23) and this conclusion was supported by molecular dynamics (MD) simulations of similar ionomers.(24) MD simulations of Li and $\mathrm{Na}$ ionic polymers with different compositions of co-cations were able to elucidate possible decoupling mechanisms and optimum compositions and sizes of the organic cation. $(25,26)$ As shown in Figure 3, when the ratio of the Li ion to the ammonium co-cation is increased to 1:1 (vs 1:5), a hopping mechanism involving rearrangement of the Li coordination environment is possible along an interconnected cluster. This is believed to be the origin of the decoupling of the alkali metal cation from the bulk polymer dynamics. The presence of an ether oxygen disturbs this connectivity and thereby shuts down the hopping in the Li case but not in the Na system. This contrast between the Liand $\mathrm{Na}$-based mixed-cation ion polymer systems demonstrates the complexity and importance of the different coordination environments for encouraging alkali metal cation transport and offers opportunities for designing materials to optimize the decoupling between the ion dynamics and the polymer dynamics. For example, instead of the hard sulfonate anion on the PAMPS backbone, a softer anion such as fluorosulfonamide may offer advantages.(27) Other anions such as borates could also be revisited as first suggested by Angell et al.(28)
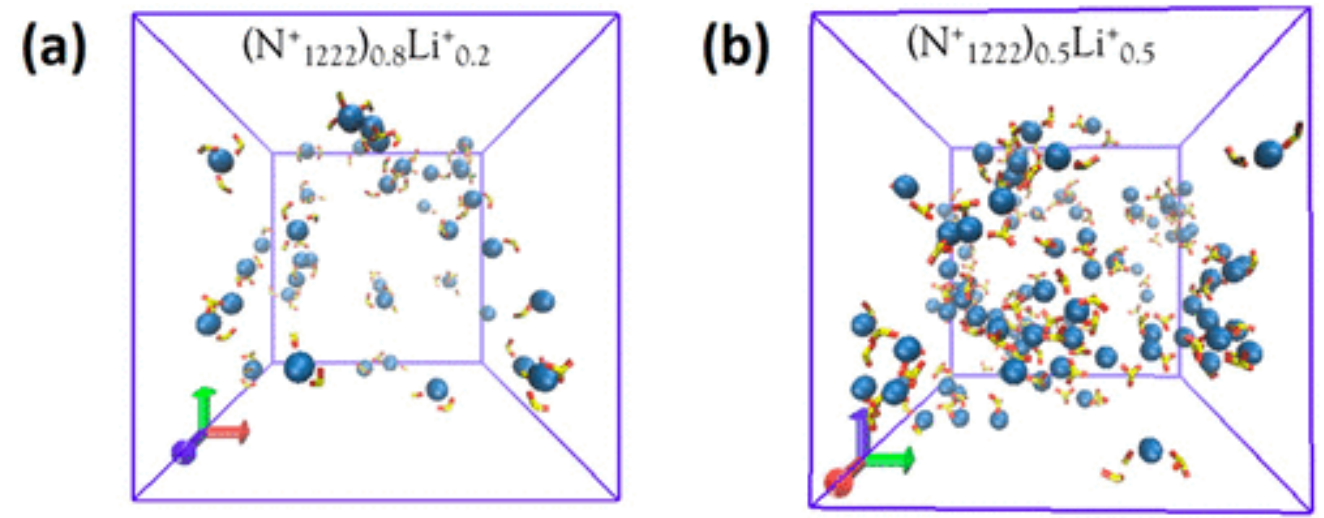

Figure 3. (a) MD simulation snapshots showing the isolated Li cluster when only $20 \%$ of cations are Li+ compared with (b) extended percolating aggregates of Li+ ions with the anions tethered to the polymer 
backbone at $50 \mathrm{~mol} \%$ of Li+ cations. Ion hopping facilitated through changing Li+ ion coordination. Yellow: sulfur; dark blue: Li+; red: oxygen; green: carbon.

Another strategy for decoupling of lithium- or sodium-ion transport from the bulk dynamics has recently been reported in both organic- and IL-based systems.(29-32) It has been demonstrated that at very high concentrations, approaching the saturation limit of the salt in the solvent, the ion speciation leads to complexes and aggregates that can percolate through the electrolyte. The ion diffusion is then supported not only by vehicular motion but by a structural diffusion mechanism similar to transport of the proton via a Grotthus mechanism in acids. MD simulations have elucidated the stark differences between the coordination environments and lithium/sodium-ion transport in the more traditional lower salt concentrations compared to the superconcentrated cases. $(33,34)$ Recent reports of Li coordination in related TFSI systems are consistent with our observations. $(35,36)$ These superconcentrated electrolytes allow dendrite-free lithium and sodium metal plating at very high current densities.(29,37,38) Furthermore, these systems have been successfully used with highenergy-density cathodes such as $\mathrm{LCO}$ and $\mathrm{NMC}(37,39-42)$ and more recently in sodium metal device [Na/IL electrolyte/NFP] cells operating at $50{ }^{\circ} \mathrm{C}$ (Figure 4$) .(43,44)$ The advantage of such electrolytes is their excellent stability such that they can be safely used at moderate temperatures, avoiding both flammability problems and electrode degradation.

(a)

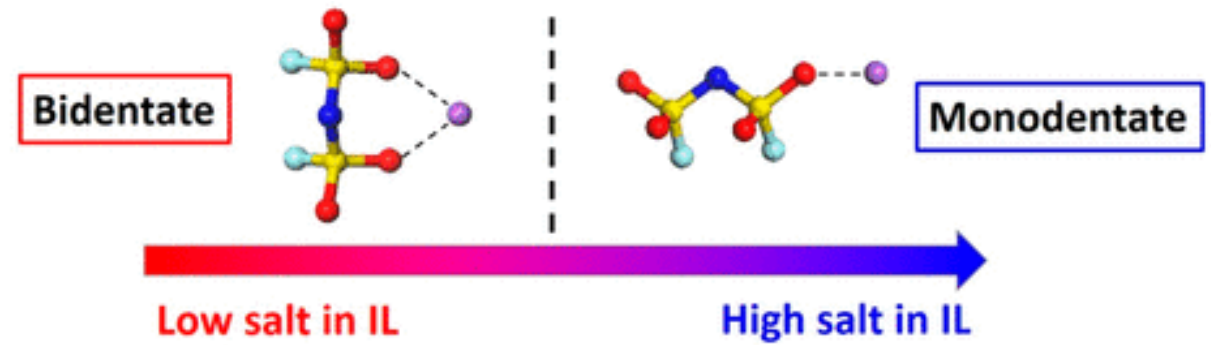

(b)

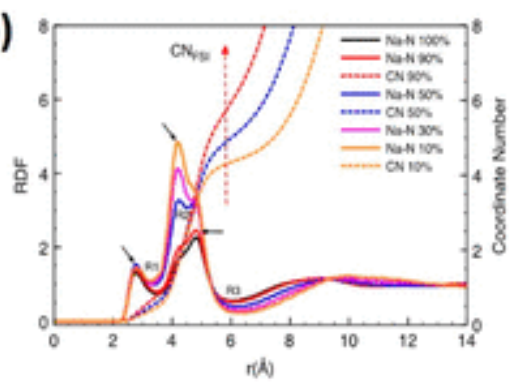

(c)

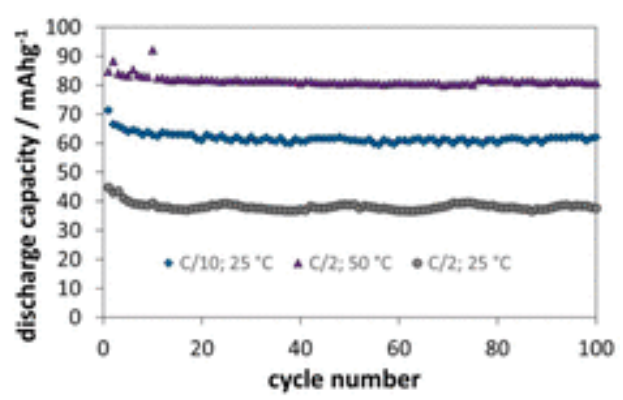

Figure 4. (a) Possible coordination environments for the Na (or Li) cation with the FSI anion with increasing salt concentration in the IL. (b) The radial distribution functions for the Na anion (in particular $\mathrm{Na}-\mathrm{N}$ ) from MD simulations as a function of salt concentration clearly showing a shift toward monodentate coordination at high salt concentrations similar to that found with the pure NaFSI salt. (c) Full cell cycling for 50 mol \% NaFSI in P111i4FSI IL electrolyte with Na/NFP electrodes under various conditions. Adapted with permission from ref (44). Copyright 2018 Elsevier.

\section{Ion Gels and Composite Electrolytes}

While superconcentrated ionic-liquid-based electrolytes clearly support stable alkali metal cycling, a key requirement for higher-energy-density batteries, they still present some challenges in a full cell, as they require a compatible separator material as mentioned above. Current separator technologies are designed for carbonate-based systems and are not necessarily applicable to these new electrolytes 
nor to higher-temperature operation. Novel separator chemistry is in of itself an important research field;(45) however, our approach has been to solidify the ionic liquid by combining it with a polymeric matrix that not only provides mechanical integrity but also may be involved in the conduction process. These materials are generally defined as "ion gels". Thus, we have focused on the ion conducting polymer material based on poly(diallyldimethylammonium) (polyDADMA) with different counteranions (polyionic liquid or "PILs") and combined with IL-based electrolytes.(46-49) We selected this polymer because of its commercial availability, low cost, high dielectric constant, and high thermal and electrochemical stability.(50)Figure 5 summarizes the chemistry of some recent examples of these materials for lithium-based electrolytes as well as for alternate chemistries such as $\mathrm{Zn}$ and Na. $(51,52)$

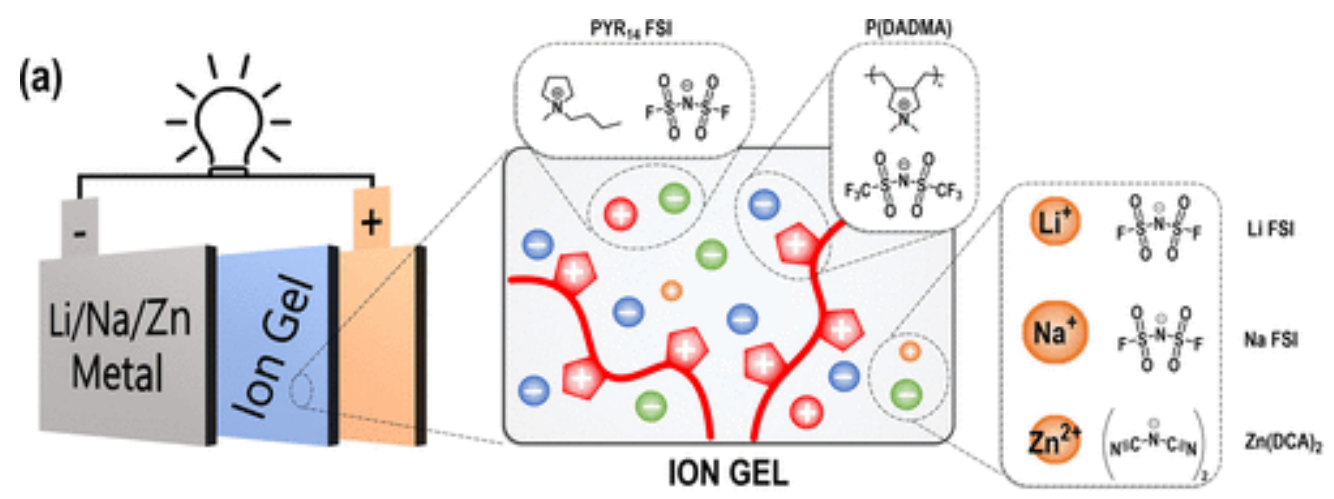

(b)

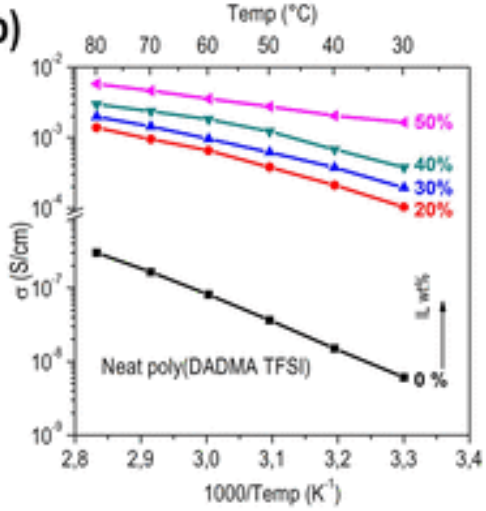

(c)

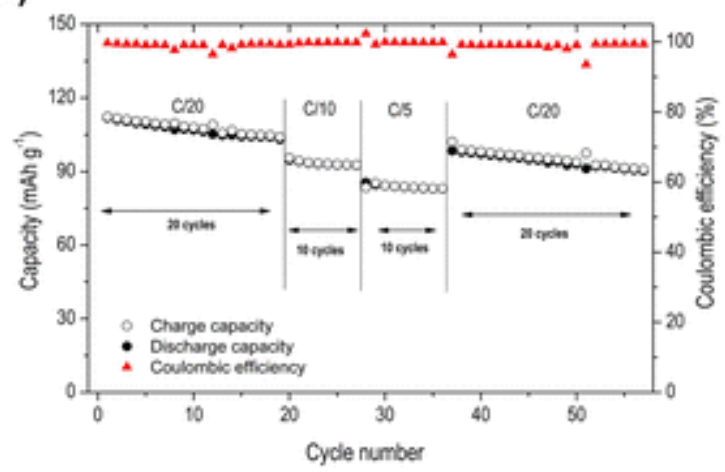

Figure 5. (a) PIL ion gels with different metal salts. These ion gels have been demonstrated in several battery chemistries as discussed in the text. (b) Conductivity of a series of $\mathrm{Na+-based} \mathrm{ion} \mathrm{gels} \mathrm{indicating}$ increasing conductivity with increasing IL content. (c) Cycling of a full Na/ion gel/NFP battery with ion gel containing $50 \%$ IL electrolyte.

As discussed above,(39-41) high-concentration IL systems have shown promising properties such as a higher lithium-ion transference number and the ability to improve battery charge-discharge performance. Recently, we have proposed a novel composite ion gel electrolyte, in which the PIL, poly(DADMA TFSI) with high molecular weight, was used as a host polymer and combined with a superconcentrated IL-based electrolyte composed of LiFSI in a phosphonium IL(46) (Figure 6b). Most importantly, diffusion coefficient measurements show that the addition of a PIL into the ionic liquids can decrease the anion diffusion more significantly compared with the lithium cations, which effectively increase the lithium-ion transport number. This could be a result of the different coordination mechanism compared with other host polymers such as PEO and polycarbonates. As also suggested by Schonhoff and co-workers,(53) our results highlight the advantages of PILs as a potential host or "solvent" for salt in polymer electrolyte materials. In PIL-based materials, the PILs can dissociate the lithium salt but have weaker coordination with the lithium cations, which benefits the 
lithium transport in contrast to PEO-based polymer electrolytes. Although the addition of ILs increases the ion conductivity of the electrolyte, the deterioration of the mechanical stability becomes an issue for this salt plasticized system. By using the same PIL but replacing the TFSI with the FSI anion, the mechanical properties can be slightly improved at a given composition.(47) Initially, it was thought that this could be due to the fact that a common anion is used in both the PIL and the IL electrolyte, which might improve compatibility; however, somewhat surprisingly, the compatibility was lesser than with the mixed anions.(47) This behavior is still not understood, and there is much room to play with different anion chemistry and anion mixtures.
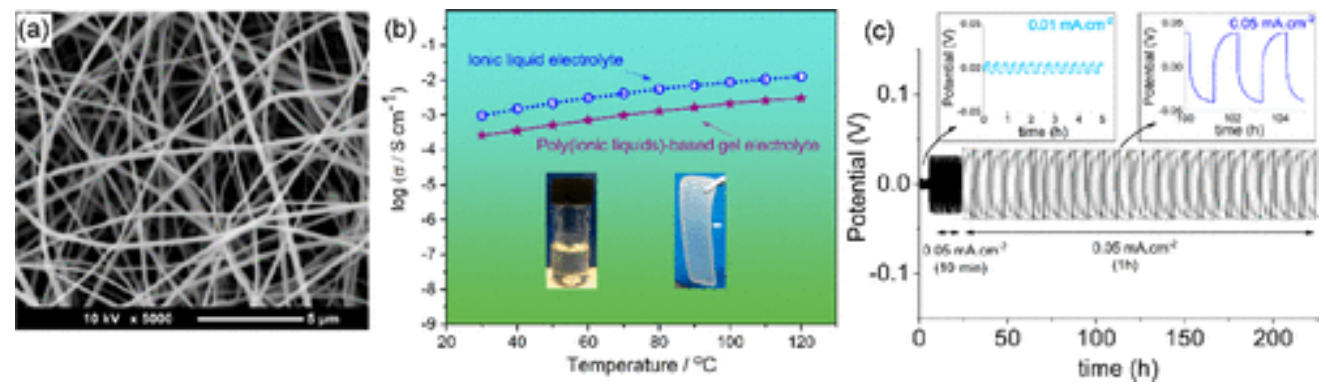

Figure 6. (a) SEM image of the electrospun nanofibers. (b) The ionic conductivity comparison between composite electrolyte (PIL/3.8 m LiFSI IL/Al2O3 = 50:50:5, by weight) and the ILs ( $3.8 \mathrm{~m} \mathrm{LiFSI} \mathrm{IL).}$ Reproduced with permission from ref (46). Copyright 2017 Royal Society of Chemistry; (c) $\mathrm{Li} /$ composite electrolyte/Li symmetric cell cycling. Data from ref (48).

More pragmatically, the addition of inorganic fillers such as Al2O3 nanoparticles is a promising way to achieve higher mechanical stability. By adding $5 \mathrm{wt} \%$ Al2O3 nanoparticles to the PIL composite systems, a higher IL loading of $60 \mathrm{wt} \%$ and a conductivity of $0.28 \mathrm{mS} \mathrm{cm}-1$ at $30{ }^{\circ} \mathrm{C}$ was recently achieved.(46) The enhancement of the mechanical properties $\left(E^{\prime}=6.4 \mathrm{MPa}\right.$ at $\left.30^{\circ} \mathrm{C}\right)$ may be attributed to the molecular entanglements between polymer chain and nanoparticles. As a result, long-term and stable symmetrical lithium cells and promising full cell charge-discharge performance have been demonstrated. $(46,49)$ However, this mechanical enhancement is still limited when more ionic liquid is added, and this can pose difficulties for large-scale devices such as pouch cells, where mechanical strength may be needed in the assembly process. An alternative method to develop a highly flexible and strong composite electrolyte was recently developed by using electrospun nanofibers composited with the PIL electrolytes to provide mechanical support.(48) Electrospinning is a robust method to prepare a fibrous matrix with high porosity (Figure 6a) and good mechanical properties. Benefiting from the improved mechanical strength of this composite electrolyte, extremely high lithium salt content has been used in the PIL-based electrolytes. Promisingly, full cell charge-discharge tests showed that these solid electrolytes operated with good stability when used with high-voltage cathode materials such as NMC and NCA, thus providing a facile new direction toward high-energydensity batteries.

Similar ion gels can be designed for alternative battery technologies to lithium. As an example, we recently designed polyDADMA ion gels for zinc and sodium batteries, respectively. In a first example, the IL 1-ethyl-3-methylimidazolium dicyanamide was combined with poly(DADMA TFSI) and Zn(dca)2 for a zinc battery.(51) The obtained electrolytes showed high ionic conductivity values $(1.1 \times 10-2$ $\mathrm{S} / \mathrm{cm}$ at $50^{\circ} \mathrm{C}$ ) together with flexible mechanical properties of the membrane. Using those ion gels, a zinc/PEDOT rechargeable battery could be assembled for the first time with higher capacity values than those of Zn/PEDOT batteries using liquid [emim][dca], thereby proving the suitability of such materials for zinc rechargeable batteries. 
In a second example, a polyDADMA ion gel was designed for a sodium-ion battery.(52) In this case, a suitable IL-sodium salt combination based on C3 mpyrFSI ionic liquid with NaFSI was chosen. The poly(DADMA TFSI) demonstrated the ability to retain up to $50 \mathrm{wt} \%$ of IL electrolyte content in a selfstanding membrane with high ionic conductivity $\left(7.1 \times 10-3 \mathrm{~S} / \mathrm{cm}\right.$ at $\left.70^{\circ} \mathrm{C}\right)$. Higher NaFSI content led to an increase of the sodium transference number but a dramatic decrease of the ionic conductivity. Some composite ion gels were also synthesized by incorporating 5 wt \% Al2O3 nanoparticles. These electrolytes also supported sodium electrochemistry as shown by excellent sodium symmetric cell cycling at $70^{\circ} \mathrm{C}$. Moreover, the optimum composition material was analyzed in a rechargeable sodium battery using $\mathrm{NaFePO} 4$ as the cathode and showing good capacity retention and Coulombic efficiencies up to $97 \%$ at $C$ rates between $C / 20$ and $C / 5$, as shown in Figure 5 above.

3.1. New Approaches To Achieve Mechanical Strength and High lonic Transport in the Same Polymer Electrolytes-Block Copolymers

More recently, attempts have been made to improve the robustness of PIL materials while retaining high conductivity by preparing block copolymers where a polystyrene block provides the mechanical strength while the PIL allows for ion transport.(54) Inspired by this chemistry, we developed novel phase separated ion gel electrolytes with high lithium transport number by combining a PIL block copolymer with a high LiFSI salt concentration and a low ionic liquid content.(55) Here, enhanced lithium transport properties were achieved by using a similar approach to that of superconcentrated ionic liquid electrolytes, where the overall anion to Li molar ratio is kept below a value of 1.5 , through the use of high LiFSI salt content. Such ion gel electrolytes operate well in LiFePO4/lithium metal cells at $50{ }^{\circ} \mathrm{C}$, with cathode loading near practical levels ( $1.8 \mathrm{mAh} \mathrm{cm}-2$, Figure 7$)$.

(a)

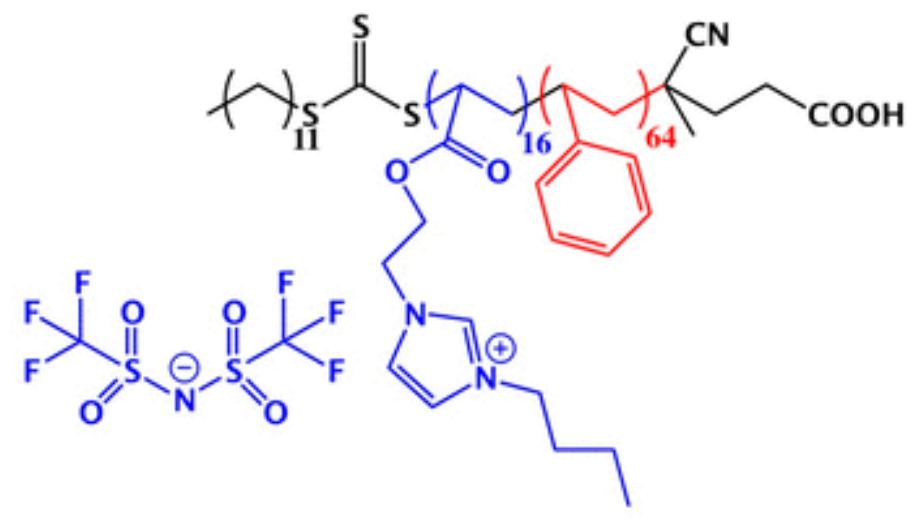

(b)

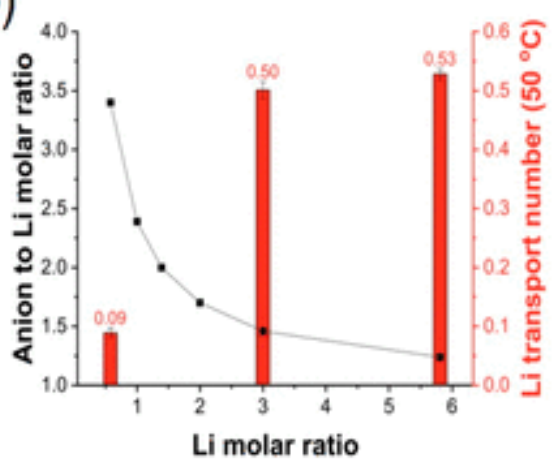

(c)

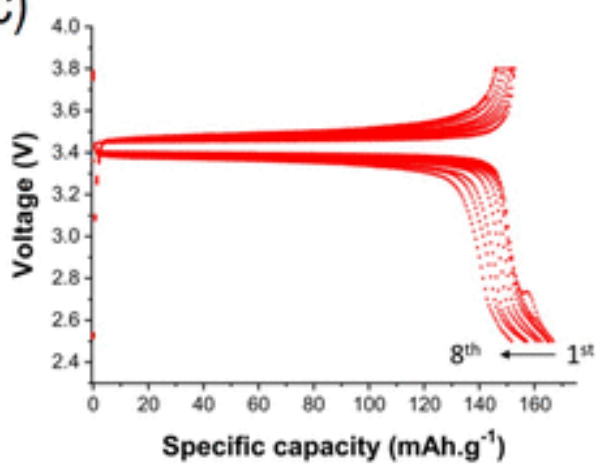

Figure 7. (a) lon gel based on LiFSI/C3mpyrFSI IL in a PS-b-PIL block copolymer. (b) Conductivity and transference number of the block copolymer ion gel. (c) Cycling of a full Na/NFP battery with a block copolymer ion gel electrolyte. Adapted with permission from ref (55). Copyright 2018 Wiley. 
A similar approach has been considered by Nguyen et al.(56) recently where an ethylene-carbonatefilled nanostructured block copolymer, consisting of polystyrene blocks and a perfluorinated sulfonamide anionic block, was demonstrated to be a safer electrolyte that performed well in a full lithium cell even when high-energy-density NCM cathodes were used. Thus, this approach of using a PIL as a host for ionic liquids and inorganic salts for a range of different battery chemistries shows significant promise both from the application perspective and with respect to new scientific understanding, since the conduction process and the role of the charged polymer backbone is still unclear.

\section{Future}

From the Account above, it is evident that there are several approaches to developing ionic polymer conductors for use in advanced energy storage technologies including lithium metal as well as alternative chemistries such as $\mathrm{Zn}$ or $\mathrm{Na}$ batteries. A polymerizable IL can be designed with alternative anion (or cation) chemistries to improve solubility or compatibility with salts and ILs. The design of anionic PILs, which can facilitate single-ion conduction, offers a wealth of opportunity, considering the idea of tethering a more delocalized anion to favor cation dissociation of the alkali metal ions. This can be coupled with our co-cation approaches where a larger organic cation can be incorporated into the backbone. Decoupling of the alkali metal cation diffusion and ionic conductivity from the polymer dynamics (i.e., Tg) has been demonstrated well using this latter approach; however, higher conductivities are required, and this may be achieved through chemical design of novel polymer backbones and the anions, as demonstrated by the recent work of Shaplov and co-workers.(57) A further dimension added by the PIL is the enhanced solubility of Li and $\mathrm{Na}$ salts in the ion gel compared to the IL system. This provides opportunities to tune the structural transport mechanism across a greater range of compositions. Although a number of different approaches to achieve single-ion conducting polymers for lithium have been investigated, the field is still in its infancy with respect to $\mathrm{Na}+$ (or other metal cations), and given the difference in size and coordination of the latter ion, there is much to explore in this case. Our preliminary work on mixed anions in ILs and our early work on zwitterion additives $(58,59)$ suggests that a conduction mechanism that favors ion hopping through structural rearrangement can be designed by invoking a mixed coordination environment around the metal ion. This is under investigation in our combined laboratories at present.

\section{Notes}

The authors declare no competing financial interest.

\section{Biographies}

Maria Forsyth

Prof. Maria Forsyth is an Alfred Deakin Professor at Deakin University, Deputy Director of the ARC Centre for Electromaterials Science, and a visiting Ikerbasque Research Professor at POLYMAT. One area of her research focuses on design, characterization, and application of novel electrolytes for advanced batteries.

Luca Porcarelli

Dr. Luca Porcarelli is a Marie Skłodowska-Curie fellow at the University of the Basque Country and is currently visiting Deakin University. His research interests focus on the applications of polymer electrolytes in energy storage and bioelectronics.

Xiaoen Wang 
Dr. Xiaoen Wang is a research fellow at Deakin University. He received his Ph.D. from Wuhan University of Technology, China. His research interests focus on solid-state polymer electrolytes, composites, and their applications in energy storage and conversation devices.

Nicolas Goujon

Dr. Nicolas Goujon studied chemistry at the University of Orléans. After receiving his Master's degree in 2011, he joined the group of Professor Maria Forsyth at Deakin University for his Ph.D. His research interests focus on nanostructured polymers for application in energy storage.

David Mecerreyes

Prof. David Mecerreyes is an Ikerbasque Research Professor at POLYMAT-University of the Basque Country. His research interests involve the synthesis of innovative polymers such as polymeric ILs for different applications including polymer electrolytes, electrochemical devices, or gas separation membranes

\section{Acknowledgments}

The authors are grateful to Prof. Michel Armand, Prof. Douglas MacFarlane, Prof. Patrick Howlett, Dr. John Chiefari, and Mr. Asier Fernandez De Añastro for helpful discussions and their assistance during the preparation of this manuscript. L.P. has received funding from the European Union's Horizon 2020 research and innovation programme under the Marie Skłodowska-Curie grant agreement No 797295. D.M. has received funding from the European Research Council under the F7P grant agreement No 306250. M.F. would like to acknowledge the financial supports through Australia-India Strategic Research Fund (AISRF 48515), ARC Australian Laureate Fellowship (grant agreement No FL110100013) and ARC Centre of Excellence for Electromaterials Science (grant agreement No CE140100012).

\section{References}

(1) Casals, L. C.; García, B. A.; Cremades, L. V. Electric Vehicle Battery Reuse: Preparing for a Second Life. J. Ind. Eng. Manag. 2017, 10, 266-285.

(2) Richa, K.; Babbitt, C. W.; Gaustad, G. Eco-Efficiency Analysis of a Lithium-Ion Battery Waste Hierarchy Inspired by Circular Economy. J. Ind. Ecol. 2017, 21, 715-730.

(3) Bachman, J. C.; Muy, S.; Grimaud, A.; Chang, H.-H.; Pour, N.; Lux, S. F.; Paschos, O.; Maglia, F.; Lupart, S.; Lamp, P.; Giordano, L.; Shao-Horn, Y. Inorganic Solid-State Electrolytes for Lithium Batteries: Mechanisms and Properties Governing Ion Conduction. Chem. Rev. 2016, 116, 140-162.

(4) Mindemark, J.; Lacey, M. J.; Bowden, T.; Brandell, D. Beyond PEO Alternative Host Materials for Li+-Conducting Solid Polymer Electrolytes. Prog. Polym. Sci. 2018, 81, 114-143.

(5) Keller, M.; Varzi, A.; Passerini, S. Hybrid Electrolytes for Lithium Metal Batteries. J. Power Sources 2018, 392, 206-225.

(6) Basile, A.; Hilder, M.; Makhlooghiazad, F.; Pozo Gonzalo, C.; MacFarlane, D. R.; Howlett, P. C.; Forsyth, M. Ionic Liquids and Organic lonic Plastic Crystals: Advanced Electrolytes for Safer High Performance Sodium Energy Storage Technologies. Adv. Energy Mater. 2018, 8, 1703491.

(7) Leng, F.; Tan, C. M.; Pecht, M. Effect of Temperature on the Aging Rate of Li Ion Battery Operating above Room Temperature. Sci. Rep. 2015, 5, 12967. 
(8) Lu, L.; Han, X.; Li, J.; Hua, J.; Ouyang, M. A Review on the Key Issues for Lithium-lon Battery Management in Electric Vehicles. J. Power Sources 2013, 226, 272-288.

(9) Armand, M.; Endres, F.; MacFarlane, D. R.; Ohno, H.; Scrosati, B. Ionic-Liquid Materials for the Electrochemical Challenges of the Future. Nat. Mater. 2009, 8, 621-629.

(10) Wright, P. V. Polymer Electrolytes the Early Days. Electro- chim. Acta 1998, 43, 1137-1143.

(11) Armand, M. The History of Polymer Electrolytes. Solid State lonics 1994, 69, 309-319.

(12) Shaplov, A. S.; Marcilla, R.; Mecerreyes, D. Recent Advances in Innovative Polymer Electrolytes Based on Poly(Ionic Liquid)S. Electrochim. Acta 2015, 175, 18-34.

(13) Ueki, T.; Watanabe, M. Macromolecules in Ionic Liquids: Progress, Challenges, and Opportunities. Macromolecules 2008, 41, 3739-3749.

(14) Zhang, H.; Li, C.; Piszcz, M.; Coya, E.; Rojo, T.; Rodriguez- Martinez, L. M.; Armand, M.; Zhou, Z. Single Lithium-Ion Conducting Solid Polymer Electrolytes: Advances and Perspectives. Chem. Soc. Rev. 2017, 46, 797-815.

(15) Porcarelli, L.; Aboudzadeh, M. A.; Rubatat, L.; Nair, J. R.; Shaplov, A. S.; Gerbaldi, C.; Mecerreyes, D. Single-Ion Triblock Copolymer Electrolytes Based on Poly(Ethylene Oxide) and Methacrylic Sulfonamide Blocks for Lithium Metal Batteries. J. Power Sources 2017, 364, 191-199.

(16) Porcarelli, L.; Shaplov, A. S.; Salsamendi, M.; Nair, J. R.; Vygodskii, Y. S.; Mecerreyes, D.; Gerbaldi, C. Single-Ion Block Copoly(Ionic Liquid)s as Electrolytes for All-Solid State Lithium Batteries. ACS Appl. Mater. Interfaces 2016, 8, 10350-10359.

(17) Porcarelli, L.; Shaplov, A. S.; Bella, F.; Nair, J. R.; Mecerreyes, D.; Gerbaldi, C. Single-lon Conducting Polymer Electrolytes for Lithium Metal Polymer Batteries That Operate at Ambient Temper- ature. ACS Energy Lett. 2016, 1, 678-682.

(18) Tudryn, G. J.; Liu, W.; Wang, S.-W.; Colby, R. H. Counterion Dynamics in Polyester-Sulfonate Ionomers with Ionic Liquid Counterions. Macromolecules 2011, 44, 3572-3582.

(19) Noor, S. A. M.; Sun, J.; MacFarlane, D. R.; Armand, M.; Gunzelmann, D.; Forsyth, M. Decoupled Ion Conduction in Poly(2- Acrylamido-2-Methyl-1-Propane-Sulfonic Acid) Homopolymers. J. Mater. Chem. A 2014, 2, 17934-17943.

(20) Mohd Noor, S. A.; Gunzelmann, D.; Sun, J.; MacFarlane, D. R.; Forsyth, M. Ion Conduction and Phase Morphology in Sulfonate Copolymer lonomers Based on lonic Liquid-Sodium Cation Mixtures. J. Mater. Chem. A 2014, 2, 365-374.

(21) Li, J.; Zhu, H.; Wang, X.; MacFarlane, D. R.; Armand, M.; Forsyth, M. Increased Ion Conduction in Dual Cation [Sodium]- [Te $t$ raal ky lammoni um] $P$ oly [ 4 - Styre nesul $f$ onyl(Trifluoromethylsulfonyl)Imide-Co-Ethylacrylate] Ionomers. J. Mater. Chem. A 2015, 3, 19989-19995.

(22) Oza, Y. V.; MacFarlane, D. R.; Forsyth, M.; O'Dell, L. A. Characterisation of Ion Transport in Sulfonate Based lonomer Systems Containing Lithium and Quaternary Ammonium Cations. Electrochim. Acta 2015, 175, 80-86.

(23) Oza, Y. V.; MacFarlane, D. R.; Forsyth, M.; O'Dell, L. A. Unexpected Effect of Tetraglyme Plasticizer on Lithium Ion Dynamics in PAMPS Based lonomers. Phys. Chem. Chem. Phys. 2016, 18, 19011-19019. 
(24) Chen, X.; Chen, F.; Forsyth, M. Molecular Dynamics Study of the Effect of Tetraglyme Plasticizer on Dual-Cation Ionomer Electrolytes. Phys. Chem. Chem. Phys. 2017, 19, 16426-16432.

(25) Chen, X.; Forsyth, M.; Chen, F. Molecular Dynamics Study of Ammonium Based Co-Cation Plasticizer Effect on Lithium Ion Dynamics in Ionomer Electrolytes. Solid State Ionics 2018, 316, 4752.

(26) Chen, X.; Chen, F.; Jońsson, E.; Forsyth, M. Molecular Dynamics Study of a Dual-Cation lonomer Electrolyte. ChemPhy- sChem 2017, 18, 230-237.

(27) Shaplov, A. S.; Vlasov, P. S.; Armand, M.; Lozinskaya, E. I.; Ponkratov, D. O.; Malyshkina, I. A.; Vidal, F.; Okatova, O. V.; Pavlov, G. M.; Wandrey, C.; Godovikov, I. A.; Vygodskii, Y. S. Design and Synthesis of New Anionic "Polymeric Ionic Liquids" with High Charge Delocalization. Polym. Chem. 2011, 2, 2609-2618.

(28) Xu, W.; Williams, M. D.; Angell, C. A. Novel Polyanionic Solid Electrolytes with Weak Coulomb Traps and Controllable Caps and Spacers. Chem. Mater. 2002, 14, 401-409.

(29) Forsyth, M.; Girard, G. M. A.; Basile, A.; Hilder, M.; MacFarlane, D. R.; Chen, F.; Howlett, P. C. Inorganic-Organic Ionic Liquid Electrolytes Enabling High Energy-Density Metal Electrodes for Energy Storage. Electrochim. Acta 2016, 220, 609-617.

(30) Wang, J.; Yamada, Y.; Sodeyama, K.; Chiang, C. H.; Tateyama, Y.; Yamada, A. Superconcentrated Electrolytes for a High-Voltage Lithium-Ion Battery. Nat. Commun. 2016, 7, 12032.

(31) Qian, J.; Henderson, W. A.; Xu, W.; Bhattacharya, P.; Engelhard, M.; Borodin, O.; Zhang, J.-G. High Rate and Stable Cycling of Lithium Metal Anode. Nat. Commun. 2015, 6, 6362.

(32) Yamada, Y.; Yamada, A. Review Superconcentrated Electro- lytes for Lithium Batteries. J. Electrochem. Soc. 2015, 162, A2406- A2423.

(33) Chen, F.; Kerr, R.; Forsyth, M. Cation Effect on Small Phosphonium Based Ionic Liquid Electrolytes with High Concen- trations of Lithium Salt. J. Chem. Phys. 2018, 148, 193813.

(34) Chen, F.; Howlett, P.; Forsyth, M. Na-Ion Solvation and High Transference Number in Superconcentrated Ionic Liquid Electrolytes: A Theoretical Approach. J. Phys. Chem. C 2018, 122, 105-114.

(35) Brinkkötter, M.; Lozinskaya, E. I.; Ponkratov, D. O.; Vlasov, P. S.; Rosenwinkel, M. P.; Malyshkina, I. A.; Vygodskii, Y.; Shaplov, A. S.; Schönhoff, M. Influence of Anion Structure on Ion Dynamics in Polymer Gel Electrolytes Composed of Poly(lonic Liquid), lonic Liquid and Li Salt. Electrochim. Acta 2017, 237, 237-247.

(36) Gouverneur, M.; Schmidt, F.; Schönhoff, M. Negative Effective Li Transference Numbers in Li Salt/lonic Liquid Mixtures: Does Li Drift in the "Wrong" Direction? Phys. Chem. Chem. Phys. 2018, 20, 7470-7478.

(37) Yoon, H.; Best, A. S.; Forsyth, M.; MacFarlane, D. R.; Howlett, P. C. Physical Properties of High Li-Ion Content N-Propyl-N- Methylpyrrolidinium Bis(Fluorosulfonyl)Imide Based Ionic Liquid Electrolytes. Phys. Chem. Chem. Phys. 2015, 17, 4656-4663.

(38) Girard, G. M. A.; Hilder, M.; Nucciarone, D.; Whitbread, K.; Zavorine, S.; Moser, M.; Forsyth, M.; MacFarlane, D. R.; Howlett, P. 
C. Role of Li Concentration and the SEI Layer in Enabling High Performance Li Metal Electrodes Using a Phosphonium Bis- (Fluorosulfonyl)Imide Ionic Liquid. J. Phys. Chem. C 2017, 121, 21087-21095.

(39) Girard, G. M. A.; Hilder, M.; Dupre, N.; Guyomard, D.; Nucciarone, D.; Whitbread, K.; Zavorine, S.; Moser, M.; Forsyth, M.; MacFarlane, D. R.; Howlett, P. C. Spectroscopic Characterization of the SEI Layer Formed on Lithium Metal Electrodes in Phosphonium Bis(Fluorosulfonyl)Imide Ionic Liquid Electrolytes. ACS Appl. Mater. Interfaces 2018, 10, 6719-6729.

(40) Hilder, M.; Girard, G. M. A.; Whitbread, K.; Zavorine, S.; Moser, M.; Nucciarone, D.; Forsyth, M.; MacFarlane, D. R.; Howlett, P. C. Physicochemical Characterization of a New Family of Small Alkyl Phosphonium Imide Ionic Liquids. Electrochim. Acta 2016, 202, 100-109.

(41) Girard, G. M. A.; Hilder, M.; Zhu, H.; Nucciarone, D.; Whitbread, K.; Zavorine, S.; Moser, M.; Forsyth, M.; MacFarlane, D. R.; Howlett, P. C. Electrochemical and Physicochemical Properties of Small Phosphonium Cation Ionic Liquid Electrolytes with High Lithium Salt Content. Phys. Chem. Chem. Phys. 2015, 17, 8706-8713.

(42) Yoon, H.; Howlett, P. C.; Best, A. S.; Forsyth, M.; MacFarlane, D. R. Fast Charge/Discharge of Li Metal Batteries Using an Ionic Liquid Electrolyte. J. Electrochem. Soc. 2013, 160, A1629-A1637.

(43) Hilder, M.; Howlett, P. C.; Saurel, D.; Gonzalo, E.; Armand, M.; Rojo, T.; Macfarlane, D. R.; Forsyth, M. Small Quaternary Alkyl Phosphonium Bis(Fluorosulfonyl)Imide lonic Liquid Electrolytes for Sodium-Ion Batteries with P2- and O3-Na2/3[Fe2/3Mn1/3]O2 Cathode Material. J. Power Sources 2017, 349, 45-51.

(44) Hilder, M.; Howlett, P. C.; Saurel, D.; Anne, H.; Casas Cabanas, M.; Armand, M.; Rojo, T.; MacFarlane, D. R.; Forsyth, M. Stable Cycling of NaFePO4 Cathodes in High Salt Concentration Ionic Liquid Electrolytes. J. Power Sources 2018, 406, 70-80.

(45) Lee, H.; Yanilmaz, M.; Toprakci, O.; Fu, K.; Zhang, X. A Review of Recent Developments in Membrane Separators for Rechargeable Lithium-Ion Batteries. Energy Environ. Sci. 2014, 7, 3857-3886.

(46) Wang, X.; Zhu, H.; Girard, G. M. A.; Yunis, R.; MacFarlane, D. R.; Mecerreyes, D.; Bhattacharyya, A. J.; Howlett, P. C.; Forsyth, M. Preparation and Characterization of Gel Polymer Electrolytes Using Poly(Ionic Liquids) and High Lithium Salt Concentration Ionic Liquids. J. Mater. Chem. A 2017, 5, 23844-23852.

(47) Yunis, R.; Girard, G. M. A.; Wang, X.; Zhu, H.; Bhattacharyya, A. J.; Howlett, P.; MacFarlane, D. R.; Forsyth, M. The Anion Effect in Ternary Electrolyte Systems Using Poly(Diallyldimethylammonium) and Phosphonium-Based Ionic Liquid with High Lithium Salt Concentration. Solid State Ionics 2018, 327, 83-92.

(48) Wang, X.; Girard, G. M. A.; Zhu, H.; Yunis, R.; MacFarlane, D. R.; Mecerreyes, D.; Bhattacharyya, A. J.; Howlett, P. C.; Forsyth, M. Flexible and Robust Poly(lonic Liquids)-Based Composite Polymer Electrolytes for High Energy Density, All-Solid-State Lithium Metal Batteries. Energy Storage Materials 2019, Submitted.

(49) Girard, G. M. A.; Wang, X.; Yunis, R.; MacFarlane, D. R.; Bhattacharyya, A. J.; Forsyth, M.; Howlett, P. C. Sustainable, Dendrite Free Lithium-Metal Electrode Cycling Achieved with Polymer Composite Electrolytes Based on a Poly(Ionic Liquid) Host. Batter. Supercaps 2019, 2, Early view. 
(50) Pont, A.-L.; Marcilla, R.; De Meatza, I.; Grande, H.; Mecerreyes, D. Pyrrolidinium-based polymeric ionic liquids as mechanically and electrochemically stable polymer electrolytes. J. Power Sources 2009, 188, 558-563.

(51) Fdz De Anastro, A.; Casado, N.; Wang, X.; Rehmen, J.; Evans, D.; Mecerreyes, D.; Forsyth, M.; Pozo-Gonzalo, C. Poly(lonic Liquid) longels for All-Solid Rechargeable Zinc/PEDOT Batteries. Electrochim. Acta 2018, 278, 271-278.

(52) Fdz De Anastro, A.; Lago, N.; Berlanga, C.; Galcerań, M.; Hilder, M.; Forsyth, M.; Mecerreyes, D. Poly(Ionic Liquid) Iongel Membranes for All Solid-State Rechargeable Sodium-lon Battery. J. Membr. Sci. 2019, Accepted.

(53) Bhandary, R.; Schönhoff, M. Polymer Effect on Lithium Ion Dynamics in Gel Polymer Electrolytes: Cationic versus Acrylate Polymer. Electrochim. Acta 2015, 174, 753-761.

(54) Choi, J.-H.; Ye, Y.; Elabd, Y. A.; Winey, K. I. Network Structure and Strong Microphase Separation for High lon Conductivity in Polymerized lonic Liquid Block Copolymers. Macromolecules 2013, 46, 5290-5300.

(55) Goujon, N.; Huynh, T.-V.; Barlow, K.; Kerr, R.; Vezzu,K.; Di Noto, V.; O’Dell, L. A.; Chiefari, J.; Howlett, P. C.; Forsyth, M. Enabling High Lithium Conductivity in Polymerized lonic Liquid Block Copolymer Electrolytes. Batter. Supercaps 2019, 2, 132-138.

(56) Nguyen, H.-D.; Kim, G.-T.; Shi, J.; Paillard, E.; Judeinstein, P.; Lyonnard, S.; Bresser, D.; lojoiu, C. Nanostructured Multi-Block Copolymer Single-Ion Conductors for Safer High-Performance Lithium Batteries. Energy Environ. Sci. 2018, 11, 3298-3309.

(57) Porcarelli, L.; Vlasov, P. S.; Ponkratov, D. O.; Lozinskaya, E. I.; Antonov, D. Y.; Nair, J. R.; Gerbaldi, C.; Mecerreyes, D.; Shaplov, A. S. Design of Ionic Liquid like Monomers towards EasyAccessible Single-Ion Conducting Polymer Electrolytes. Eur. Polym. J. 2018, 107, 218-228.

(58) Byrne, N.; Pringle, J. M.; Tiyapiboonchaiya, C.; MacFarlane, D. R.; Forsyth, M. The Additive Effect of Zwitterion and Nano-Particles on Ion Dissociation in Polyelectrolytes. Electrochim. Acta 2005, $50,2733-2738$.

(59) Tiyapiboonchaiya, C.; Pringle, J. M.; Sun, J.; Byrne, N.; Howlett, P. C.; MacFarlane, D. R.; Forsyth, M. The Zwitterion Effect in High-Conductivity Polyelectrolyte Materials. Nat. Mater. 2004, 3, 29-32. 